\title{
THE CHALLENGES OF INTEGRATING INSTRUMENTATION WITH INFLATABLE AERODYNAMIC DECELERATORS
}

\author{
Gregory T. Swanson ${ }^{1}$, Alan M. Cassell ${ }^{2}$ \\ ${ }^{1}$ [ERC Incorporated], Entry Systems and Vehicle Development Branch, NASA Ames Research Center, Moffett Field, \\ CA94035 USA Email:gregory.t.swanson@nasa.gov \\ ${ }^{2}$ NASA Ames Research Center, Moffett Field, CA 94035 USA Email:alan.m.cassell@nasa.gov
}

\begin{abstract}
To realize the National Aeronautics and Space Administration's (NASA) goal of landing humans on Mars, development of technologies to facilitate the landing of heavy payloads are being explored. Current entry, decent, and landing technologies are not practical when utilizing these heavy payloads due to mass and volume constraints dictated by limitations imposed by current launch vehicle fairings. Therefore, past and present technologies are now being considered to provide a mass and volume efficient solution, including Inflatable Aerodynamic Decelerators (IADs) [1]. IAD ground and flight tests are currently being conducted to develop and characterize their performance under flight-like conditions. The integrated instrumentation systems, which are key to the performance characterization in each of these tests, have proven to be a challenge compared to the instrumentation of traditional rigid aeroshells. To overcome these challenges, flexible and embedded sensing systems have been developed, along with improved instrumenting techniques. This development opportunity faces many difficult aspects specific to inflatable structures in extreme environments. These include but are not limited to: physical flexibility, packaging, temperature, structural integration and data acquisition [2].

To better define the instrumentation challenges posed by IAD technology development, a survey was conducted to identify valuable measurements for ground and flight testing. From this survey many sensing technologies were explored, resulting in a down-selection to the most viable prospects. These systems were then iterated upon in design to determine the best integration techniques specific to a $3 \mathrm{~m}$ and $6 \mathrm{~m}$ stacked torus IAD. Each sensing system was then integrated and employed to support the IAD testing in the National Full-Scale Aerodynamics Complex 40' $x$ 80' wind tunnel at NASA Ames Research Center in the summer of 2012.

Another challenge that has been explored is the data acquisition of IAD sensing technologies. Traditionally all space based sensing systems transmit their data through a wired interface. This limits the amount of sensors able to be integrated within the IAD due to volume and routing restrictions of the supporting signal and excitation wires. To alleviate this situation, multiple wireless data acquisition technologies have been researched and developed through rapid prototyping efforts. The final custom multi-nodal wireless system utilized during the summer 2012 IAD test series consisted of four remote nodes and one receiving base station. The system reliably conditioned and acquired 20+ sensors over the course of the wind tunnel test series. These developments in wireless data acquisition techniques can eliminate the need for structural feedthroughs and reduce system mass associated with wiring and wire harnesses. This makes the utilization of flight instrumentation more attractive to future missions, which would result in further improved characterization of IAD technology, and overall, increased scientific knowledge regarding the response of inflatable structures to extreme entry environments.
\end{abstract}

[1] Clark, I.G., Hutchings, A.L., Tanner, C.L., Braun, R.D., "Supersonic Inflatable Aerodynamic Decelerators for Use on Future Robotic Missions to Mars," Journal of Spacecraft and Rockets, Vol. 46, No. 2, March 2009.

[2] Brandon, E.J. et al., Structural Health Management Technologies for Inflatable / Deployable Structures: Integrated Sensing and SelfHealing, Acta Astronautica (2010), doi:10.1016/j.actaastro.2010.08.016 